\title{
Tunable multiwavelength Brillouin-Erbium fiber laser with intra-cavity pre-amplified Brillouin pump
}

\begin{abstract}
We have demonstrated a new configuration of Brillouin-Erbium fiber laser, in which the Brillouin pump is preamplified within the laser cavity before entering the single-mode fiber. By using this simple scheme, a lower external Brillouin pump power is required to create the Brillouin gain and suppresses the laser cavity modes. The proposed laser structure exhibits a wide tuning range of $13 \mathrm{~nm}$ from $1597 \mathrm{~nm}$ to $1610 \mathrm{~nm}$ with $1480 \mathrm{~nm}$ pump power of 100 $\mathrm{mW}$. The number of channels obtained within this wavelength range is 14 channels with $0.089 \mathrm{~nm}$ spacing.
\end{abstract}

Keyword: multiwavelength fiber laser, optical fiber devices, nonlinear-Brillouin scattering 